\title{
Strong differential subordination and superordination of analytic functions in connection with a linear operator
}

\author{
T. K. Suresh ${ }^{1 *}$, D. Madhusudana Reddy ${ }^{2}$ and E. Keshava Reddy ${ }^{3}$
}

\begin{abstract}
The properties of Strong differential subordination and superordination are determined on a linear operator for some families analytic functions in the open unit disk by investigating appropriate classes of admissible functions. New sandwich-type of results for strong differential are also obtained on the linear operator.

Keywords

Strong Differential Subordination, Strong Differential Superordination, Analytic Functions, Admissible Functions, Hadamard Product.

\section{AMS Subject Classification}

30C80, 30C45.

${ }^{1}$ Department of Mathematics, Easwari Engineering College, Chennai - 600 089, Tamilnadu, India.

${ }^{2,3}$ Department of Mathematics, Jawaharlal Nehru Technological University Anantapur, Anantapuramu - 515 002, Andhra Pradesh, India.

${ }^{*}$ Corresponding author: ${ }^{1}$ tksuresh73@yahoo.com

Article History: Received 22 November 2018; Accepted 09 May 2019

(C)2018 MJM
\end{abstract}

\section{Contents}

1 Introduction 263

2 The main subordination results. 265

3 Superordination and Sandwich-type Results .....267

References .269

\section{Introduction}

Let $\mathscr{H}(\mathbb{U})$ denote the class of analytic functions in the open unit disk $\mathbb{U}:=\{z \in \mathbb{C}:|z|<1\}$. For $n \in \mathbb{N}=\{1,2,3, \ldots\}$ and $a \in \mathbb{C}$, let

$\mathscr{H}[a, n]=\left\{f: f \in \mathscr{H}(\mathbb{U}), f(z)=a+a_{n} z^{n}+a_{n+1} z^{n+1}+\cdots\right\}$

, with $\mathscr{H}_{0} \equiv \mathscr{H}[0,1]$ and $\mathscr{H} \equiv \mathscr{H}[1,1]$. Let $\mathscr{A}$ denote the class of all normalized analytic functions of the form

$$
f(z)=z+\sum_{n=2}^{\infty} a_{n} z^{n} \quad(z \in \mathbb{U}) .
$$

Let $f$ and $F$ be members of $\mathscr{H}(\mathbb{U})$. The function $f$ is said to be subordinate to $F$, or (equivalently) $F$ is said to be superordinate to $f$, if there exists a Schwarz function $w$ analytic in $\mathbb{U}$, with $w(0)=0$ and $|w(z)|<1$, such that
$f(z)=F(w(z))$. In such a case, we write $f \prec F$ or $f(z) \prec$ $F(z)$. If the function $F$ is univalent in $\mathbb{U}$, then we have

$$
f \prec F \quad \Longleftrightarrow \quad f(0)=F(0) \quad \text { and } \quad f(\mathbb{U}) \subset F(\mathbb{U}) .
$$

Let $H(z, \zeta)$ be analytic in $\mathbb{U} \times \overline{\mathbb{U}}$ and let $f(z)$ be analytic and univalent in $\mathbb{U}$. Then the function $H(z, \zeta)$ is said to be strongly subordinate to $f(z)$, or $f(z)$ is said to be strongly superordinate to $H(z, \zeta)$, written as $H(z, \zeta) \prec \prec f(z)$, if, for $\zeta \in \overline{\mathbb{U}}, H(z, \zeta)$ as a function of $z$ is subordinate to $f(z)$. We note that

$H(z, \zeta) \prec \prec f(z) \Longleftrightarrow H(0, \zeta)=f(0)$ and $H(\mathbb{U} \times \overline{\mathbb{U}}) \subset f(\mathbb{U})$

For a function $f$ given by (1.1) and $g$ given by

$$
g(z)=z+\sum_{n=2}^{\infty} b_{n} z^{n}
$$

we denote by $(f * g)(z)$ the Hadamard product (or convolution) of $f$ and $g$, defined by

$$
(f * g)(z):=z+\sum_{n=2}^{\infty} a_{n} b_{n} z^{n}:=(g * f)(z) .
$$

For $\alpha_{j} \in \mathbb{C}(j=1,2, \ldots q)$ and $\beta_{k} \in \mathbb{C} \backslash\{0,-1,-2, \ldots\}$ $(k=1,2, \ldots, s)$ the generalized hypergeometric function 
${ }_{q} F_{s}\left(\alpha_{1}, \ldots, \alpha_{q} ; \beta_{1}, \ldots, \beta_{s} ; z\right)$ is defined by the infinite series

$$
\begin{aligned}
& { }_{q} F_{s}\left(\alpha_{1}, \ldots, \alpha_{q} ; \beta_{1}, \ldots, \beta_{s} ; z\right):=\sum_{n=0}^{\infty} \frac{\left(\alpha_{1}\right)_{n} \ldots\left(\alpha_{q}\right)_{n}}{\left(\beta_{1}\right)_{n} \ldots\left(\beta_{s}\right)_{n}} \frac{z^{n}}{n !} \\
& \left(q \leq s+1, q, s \in \mathbb{N}_{0}:=\mathbb{N} \cup\{0\}\right),
\end{aligned}
$$

where $(a)_{n}$ is the Pochhammer symbol (or the shifted factorial) given by

$(a)_{n}:= \begin{cases}1 & n=0 ; a \in \mathbb{C} \backslash\{0\}, \\ a(a+1)(a+2) \ldots(a+n-1) & n \in \mathbb{N} ; a \in \mathbb{C} .\end{cases}$

Corresponding to the function

$$
F\left(\alpha_{1}, \ldots, \alpha_{q} ; \beta_{1}, \ldots, \beta_{s} ; z\right)=z_{q} F_{s}\left(\alpha_{1}, \ldots, \alpha_{q} ; \beta_{1}, \ldots, \beta_{s} ; z\right)
$$

Kwon and Cho [14] recently introduced a function $\mathscr{F}_{\lambda}\left(\alpha_{1}, \ldots, \alpha_{q} ; \beta_{1}, \ldots, \beta_{s} ; z\right)$ given by $F\left(\alpha_{1}, \ldots, \alpha_{q} ; \beta_{1}, \ldots, \beta_{s} ; z\right) * \mathscr{F}_{\lambda}\left(\alpha_{1}, \ldots, \alpha_{q} ; \beta_{1}, \ldots, \beta_{s} ; z\right)$

$$
=\frac{z}{(1-z)^{\lambda}} \quad(\lambda>0) \text {. }
$$

Analogous to Dziok-Srivastava operator, Kwon and Cho [14], introduced and investigated the linear operator

$$
H_{\lambda}\left(\alpha_{1}, \ldots, \alpha_{q} ; \beta_{1}, \ldots, \beta_{s}\right): \mathscr{A} \longrightarrow \mathscr{A}
$$

defined in terms of the Hadamard product (or convolution) by $H_{\lambda}\left(\alpha_{1}, \ldots, \alpha_{q} ; \beta_{1}, \ldots, \beta_{s} ; z\right) f(z)$

$$
=\mathscr{F}_{\lambda}\left(\alpha_{1}, \ldots, \alpha_{q} ; \beta_{1}, \ldots, \beta_{s} ; z\right) * f(z)
$$

$$
\left(\alpha_{i}, \beta_{j} \in \mathbb{C} \backslash \mathbb{Z}_{0}^{-} ; i=1, \ldots, q ; j=1, \ldots, s, \lambda>0, f \in \mathscr{A}\right) .
$$

It is easy to observe from (1.4) and (1.5) that

$$
\begin{aligned}
& H_{\lambda}\left(\alpha_{1}, \ldots, \alpha_{q} ; \beta_{1}, \ldots, \beta_{s} ; z\right) f(z) \\
& =z+\sum_{n=2}^{\infty} \frac{\left(\beta_{1}\right)_{n-1} \ldots\left(\beta_{s}\right)_{n-1}}{\left(\alpha_{1}\right)_{n-1} \ldots\left(\alpha_{q}\right)_{n-1}}(\lambda)_{n-1} a_{n} z^{n}
\end{aligned}
$$

For convenience, we write $H_{\lambda, q, s}\left(\alpha_{1}\right):=H_{\lambda}\left(\alpha_{1}, \ldots, \alpha_{q} ; \beta_{1}, \ldots, \beta_{s}\right)$. It is easily verified from (1.6) that

$$
\begin{aligned}
& z\left(H_{\lambda, q, s}\left(\alpha_{1}+1\right) f\right)^{\prime}(z) \\
& \quad=\alpha_{1} H_{\lambda, q, s}\left(\alpha_{1}\right) f(z)-\left(\alpha_{1}-1\right) H_{\lambda, q, s}\left(\alpha_{1}+1\right) f(z) .
\end{aligned}
$$

In particular, the operator $H_{\lambda, 2,1}(\gamma+1,1 ; 1)(\lambda>0 ; \gamma>-1)$ were introduced by Choi et al. [4]. For $\gamma=n$ and $\lambda=2$, the operator $H_{2,2,1}(n+1,1 ; 1)$ is the Noor Integral operator of $n$th order of $f$ studied in [8].

Definition 1.1. [13] Let $\phi: \mathbb{C}^{3} \times \mathbb{U} \times \overline{\mathbb{U}} \rightarrow \mathbb{C}$ and let $h(z)$ be univalent in $\mathbb{U}$. If $p(z)$ is analytic in $\mathbb{U}$ and satisfies the following (second-order) strong differential subordination:

$$
\phi\left(p(z), z p^{\prime}(z), z^{2} p^{\prime \prime}(z) ; z, \zeta\right) \prec \prec h(z),
$$

then $p(z)$ is called a solution of the strong differential subordination. The univalent function $q(z)$ is called a dominant of the solutions of the strong differential subordination or more simply a dominant if $p(z) \prec q(z)$ for all $p(z)$ satisfying (1.8). A dominant $\tilde{q}(z)$ that satisfies $\tilde{q}(z) \prec q(z)$ for all dominants $q(z)$ of (1.8) is said to be the best dominant.

Recently, Oros [10] introduced the following notion of strong differential superordinations as the dual concept of strong differential subordinations.

Definition 1.2. $[7,10]$ Let $\varphi: \mathbb{C}^{3} \times \mathbb{U} \times \overline{\mathbb{U}} \rightarrow \mathbb{C}$ and let $h(z)$ be analytic in $\mathbb{U}$. If $p(z)$ and $\varphi\left(p(z), z p^{\prime}(z), z^{2} p^{\prime \prime}(z) ; z, \zeta\right)$ are univalent in $\mathbb{U}$ for $\zeta \in \overline{\mathbb{U}}$ and satisfy the following (secondorder) strong differential superordination:

$$
h(z) \prec \prec \varphi\left(p(z), z p^{\prime}(z), z^{2} p^{\prime \prime}(z) ; z, \zeta\right),
$$

then $p(z)$ is called a solution of the strong differential superordination. An analytic function $q(z)$ is called a subordinant of the solution of the strong differential superordination or more simply a subordinant if $q(z) \prec p(z)$ for all $p(z)$ satisfying (1.9). A univalent subordinant $\tilde{q}(z)$ that satisfies $q(z) \prec \tilde{q}(z)$ for all subordinants $q(z)$ of (1.9) is said to be the best subordinant.

We denote by $\mathscr{Q}$ the class of functions $q$ that are analytic and injective on $\overline{\mathbb{U}} \backslash E(q)$, where

$E(q)=\left\{\xi \in \partial \mathbb{U}: \lim _{z \rightarrow \xi} q(z)=\infty\right\}$, and are such that $q^{\prime}(\xi) \neq 0$ for $\xi \in \partial \mathbb{U} \backslash E(q)$. Further, let the subclass of $\mathscr{Q}$ for which $q(0)=a$ be denoted by $\mathscr{Q}(a), \mathscr{Q}(0) \equiv \mathscr{Q}_{0}$ and $\mathscr{Q}(1) \equiv \mathscr{Q}_{1}$.

Definition 1.3. [13] Let $\Omega$ be a set in $\mathbb{C}, q \in \mathscr{Q}$ and $n \in \mathbb{N}$. The class of admissible functions $\Psi_{n}[\Omega, q]$ consists of those functions $\psi: \mathbb{C}^{3} \times \mathbb{U} \times \overline{\mathbb{U}} \rightarrow \mathbb{C}$ that satisfy the following admissibility condition: $\psi(r, s, t ; z, \zeta) \notin \Omega$ whenever $r=q(\xi)$, $s=k \xi q^{\prime}(\xi)$ and $\Re\left(\frac{t}{s}+1\right) \geq k \Re\left\{\frac{\xi q^{\prime \prime}(\xi)}{q^{\prime}(\xi)}+1\right\},(z \in \mathbb{U} ; \xi \in$ $\partial \mathbb{U} \backslash E(q) ; \zeta \in \bar{U} ; k \geq n)$. We simply write $\Psi_{1}[\Omega, q]$ as $\Psi[\Omega, q]$.

Definition 1.4. [10] Let $\Omega$ be a set in $\mathbb{C}$ and $q \in \mathscr{H}[a, n]$ with $q^{\prime}(z) \neq 0$. The class of admissible functions $\Psi_{n}^{\prime}[\Omega, q]$ consists of those functions $\psi: \mathbb{C}^{3} \times \mathbb{U} \times \overline{\mathbb{U}} \rightarrow \mathbb{C}$ that satisfy the following admissibility condition: $\psi(r, s, t ; \xi, \zeta) \in \Omega$ whenever $r=q(z), \quad s=\frac{z q^{\prime}(z)}{m}, \quad$ and $\mathfrak{R}\left(\frac{t}{s}+1\right) \leq \frac{1}{m} \mathfrak{R}\left\{\frac{z q^{\prime \prime}(z)}{q^{\prime}(z)}+1\right\}, \quad(z \in \mathbb{U} ; \xi \in \partial \mathbb{U} ; \zeta \in \overline{\mathbb{U}} ;$ $m \geq n \geq 1)$. In particular, we write $\Psi_{1}^{\prime}[\Omega, q]$ as $\Psi^{\prime}[\Omega, q]$.

For the above two classes of admissible functions, G.I. Oros and G. Oros [13] proved the following result.

Lemma 1.5. [13] Let $\psi \in \Psi_{n}[\Omega, q]$ with $q(0)=a$. If $p \in \mathscr{H}[a, n]$ satisfies $\psi\left(p(z), z p^{\prime}(z), z^{2} p^{\prime \prime}(z) ; z, \zeta\right) \in \Omega$, then $p(z) \prec q(z) \quad(z \in \mathbb{U})$.

G.I. Oros [10], on the other hand proved Lemma 1.6. 
Lemma 1.6. [10] Let $\psi \in \Psi_{n}^{\prime}[\Omega, q]$ with $q(0)=a$. If $p \in$ $\mathscr{Q}(a)$ and $\psi\left(p(z), z p^{\prime}(z), z^{2} p^{\prime \prime}(z) ; z, \zeta\right)$ is univalent in $\mathbb{U}$ for $\zeta \in \overline{\mathbb{U}}$, then $\Omega \subset\left\{\psi\left(p(z), z p^{\prime}(z), z^{2} p^{\prime \prime}(z) ; z, \zeta\right): z \in \mathbb{U}, \zeta \in \overline{\mathbb{U}}\right\}$ implies the following subordination relationship:

$q(z) \prec p(z) \quad(z \in \mathbb{U})$.

In this present investigation, by making use of results of Oros and Oros [10,13], we consider certain suitable classes of admissible functions and investigate some strong differential subordination and strong differential superordination properties of analytic functions associated with the Kwon-Cho linear operator $H_{\lambda, q, s}\left(\alpha_{1}\right)$ defined by (1.6). New strong differential sandwich-type results associated with the Kwon-Cho linear operator are also obtained. Recently, there are many interesting results obtained by several authors in strong differential subordination and superordination [1-3, 5, 9-13].

\section{The main subordination results}

We first define the following class of admissible functions that are required in the first result.

Definition 2.1. Let $\Omega$ be a set in $\mathbb{C}$ and $q \in \mathscr{Q}_{1} \mathscr{A} p \mathscr{H}$. The class of admissible function $\Phi_{H}[\Omega, q]$ consists of those function $\phi: \mathbb{C}^{3} \times \mathbb{U} \times \overline{\mathbb{U}} \rightarrow \mathbb{C}$ that satisfy the admissibility condition: $\quad \phi(u, v, w ; z, \zeta) \notin \Omega$ whenever $u=q(\xi)$, $v=\frac{k \xi q^{\prime}(\xi)+\left(\alpha_{1}-2\right) q(\xi)}{\left(\alpha_{1}-1\right)}$, and

$\Re\left\{\frac{\left(\alpha_{1}-1\right)\left(\alpha_{2}-1\right) w-\left(\alpha_{1}-2\right)\left(\alpha_{1}-3\right) u}{\left(\alpha_{1}-1\right) v-\left(\alpha_{1}-2\right) u}-\alpha_{1}+5\right\} \geq k \Re\left\{\frac{\xi q^{\prime \prime}(\xi)}{q^{\prime}(\xi)}+1\right\}$, $(z \in \mathbb{U} ; \xi \in \partial \mathbb{U} \backslash E(q) ; \zeta \in \overline{\mathbb{U}} ; k \geq 1)$.

Theorem 2.2. Let $\phi \in \Phi_{H}[\Omega, q]$. If $f \in \mathscr{A}$ satisfies

$$
\begin{aligned}
& \left\{\phi \left(H_{\lambda, q, s}\left(\alpha_{1}\right) f(z), H_{\lambda, q, s}\left(\alpha_{1}-1\right) f(z),\right.\right. \\
& \left.\left.H_{\lambda, q, s}\left(\alpha_{1}-2\right) f(z) ; z, \zeta\right): z \in \mathbb{U}, \zeta \in \overline{\mathbb{U}}\right\} \subset \Omega,
\end{aligned}
$$

then $H_{\lambda, q, s}\left(\alpha_{1}\right) f(z) \prec q(z)$.

Proof. Define the function $p$ in $\mathbb{U}$ by

$$
p(z):=H_{\lambda, q, s}\left(\alpha_{1}\right) f(z) .
$$

A simple calculation yields

$$
H_{\lambda, q, s}\left(\alpha_{1}-1\right) f(z)=\frac{1}{\alpha_{1}-1}\left[\left(\alpha_{1}-2\right) p(z)+z p^{\prime}(z)\right] .
$$

Further computations show that

$$
\begin{aligned}
& H_{\lambda, q, s}\left(\alpha_{1}-2\right) f(z) \\
& =\frac{\left(\alpha_{1}-2\right)\left(\alpha_{1}-3\right) p(z)+\left(\alpha_{1}-4\right) z p^{\prime}(z)+z^{2} p^{\prime \prime}(z)}{\left(\alpha_{1}-1\right)\left(\alpha_{2}-1\right)} .
\end{aligned}
$$

We now define the transformations from $\mathbb{C}^{3}$ to $\mathbb{C}$ by

$$
\begin{aligned}
& u=r, v=\frac{\left(\alpha_{1}-2\right) r+s}{\alpha_{1}-1}, \\
& w=\frac{\left(\alpha_{1}-2\right)\left(\alpha_{1}-3\right) r+\left(\alpha_{1}-4\right) s+t}{\left(\alpha_{1}-1\right)\left(\alpha_{2}-1\right)} .
\end{aligned}
$$

Let $\psi(r, s, t ; z, \zeta)=\phi(u, v, w ; z, \zeta)$

$$
\begin{aligned}
=\phi\left(r, \frac{s+\left(\alpha_{1}-2\right) r}{\alpha_{1}-1},\right. \\
\\
\left.\quad \frac{t+\left(\alpha_{1}-4\right) s+\left(\alpha_{1}-2\right)\left(\alpha_{1}-3\right) r}{\left(\alpha_{1}-1\right)\left(\alpha_{2}-1\right)} ; z, \zeta\right) .
\end{aligned}
$$

The proof will make use of Lemma 1.5. Using (2.2), (2.3), and (2.4), from (2.6) we obtain

$$
\begin{aligned}
& \psi\left(p(z), z p^{\prime}(z), z^{2} p^{\prime \prime}(z) ; z, \zeta\right) \\
&=\phi\left(H_{\lambda, q, s}\left(\alpha_{1}\right) f(z), H_{\lambda, q, s}\left(\alpha_{1}-1\right) f(z),\right. \\
&\left.H_{\lambda, q, s}\left(\alpha_{1}-2\right) f(z) ; z, \zeta\right) .
\end{aligned}
$$

Hence (2.1) becomes $\psi\left(p(z), z p^{\prime}(z), z^{2} p^{\prime \prime}(z) ; z, \zeta\right) \in \Omega$. A computation using (2.5) yields

$$
\frac{t}{s}+1=\frac{\left(\alpha_{1}-1\right)\left(\alpha_{2}-1\right) w-\left(\alpha_{1}-2\right)\left(\alpha_{1}-3\right) u}{\left(\alpha_{1}-1\right) v-\left(\alpha_{1}-2\right) u}-\alpha_{1}+5 .
$$

Thus the admissibility condition for $\phi \in \Phi_{H}[\Omega, q]$ in Definition 2.1 is equivalent to the admissibility condition for $\psi$ as given in Definition 1.3. Hence $\psi \in \Psi[\Omega, q]$ and by Lemma $1.5 p(z) \prec q(z)$ or, equivalently,

$H_{\lambda, q, s}\left(\alpha_{1}\right) f(z) \prec q(z)$, which evidently completes the proof of Theorem 2.2.

If $\Omega \neq \mathbb{C}$ is a simply connected domain, then $\Omega=h(\mathbb{U})$ for some conformal mapping $h$ of $\mathbb{U}$ onto $\Omega$. In this case, the class $\Phi_{H}[h(\mathbb{U}), q]$ is written as $\Phi_{H}[h, q]$. The following result is an immediate consequence of Theorem 2.2.

Theorem 2.3. Let $\phi \in \Phi_{H}[h, q]$. If $f \in \mathscr{A}$ satisfies

$$
\begin{aligned}
& \phi\left(H_{\lambda, q, s}\left(\alpha_{1}\right) f(z), H_{\lambda, q, s}\left(\alpha_{1}-1\right) f(z),\right. \\
&\left.H_{\lambda, q, s}\left(\alpha_{1}-2\right) f(z) ; z, \zeta\right) \prec \prec h(z),
\end{aligned}
$$

then $H_{\lambda, q, s}\left(\alpha_{1}\right) f(z) \prec q(z)$.

Our next result in an extension of Theorem 2.2 to the case in which the behavior of $q$ on $\partial \mathbb{U}$ is not known.

Theorem 2.4. Let $h$ and $q$ be univalent in $\mathbb{U}$ with $q(0)=0$, and set $q_{\rho}(z)=q(\rho z)$ and $h_{\rho}(z)=h(\rho z)$. Let $\phi: \mathbb{C}^{3} \times \mathbb{U} \times$ $\overline{\mathbb{U}} \rightarrow \mathbb{C}$ satisfies one of the following conditions:

(i) $\phi \in \Phi_{H}\left[h, q_{\rho}\right]$ for some $\rho \in(0,1)$, or

(ii) there exist $\rho_{0} \in(0,1)$ such that $\phi \in \Phi_{H}\left[h_{\rho}, q_{\rho}\right]$ for all $\rho \in\left(\rho_{0}, 1\right)$.

If $f \in \mathscr{A}$ satisfies (2.8), then $H_{\lambda, q, s}\left(\alpha_{1}\right) f(z) \prec q(z)$.

Proof. The proof of Theorem 2.4 is similar to that of a known result [6, Definition 2.3d, page 30] and so it is omitted here.

Our next theorem produces the best dominant of the strong differential subordination (2.8). 
Theorem 2.5. Let $h$ be univalent in $\mathbb{U}$, and $\phi: \mathbb{C}^{3} \times \mathbb{U} \times \overline{\mathbb{U}} \rightarrow$ $\mathbb{C}$. Suppose that the following differential equation:

$$
\begin{aligned}
& \phi\left(q(z), \frac{\left(\alpha_{1}-2\right) q(z)+z q^{\prime}(z)}{\alpha_{1}-1},\right. \\
& \left.\quad \frac{\left(\alpha_{1}-2\right)\left(\alpha_{1}-3\right) q(z)+\left(\alpha_{1}-4\right) z q^{\prime}(z)+z^{2} q^{\prime \prime}(z)}{\left(\alpha_{1}-1\right)\left(\alpha_{2}-1\right)} ; z, \zeta\right) \\
& \quad=h(z)
\end{aligned}
$$

has a solution $q$ with $q(0)=0$ and satisfies one of the following conditions:

(i) $q \in \mathscr{Q}_{0}$ and $\phi \in \Phi_{H}[h, q]$,

(ii) $q$ is univalent in $\mathbb{U}$ and $\phi \in \Phi_{H}\left[h, q_{\rho}\right]$ for some $\rho \in$ $(0,1)$, or

(iii) $q$ is univalent in $\mathbb{U}$ and there exists $\rho_{0} \in(0,1)$ such that $\phi \in \Phi_{H}\left[h_{\rho}, q_{\rho}\right]$ for all $\rho \in\left(\rho_{0}, 1\right)$.

If $f \in \mathscr{A}$ satisfies (2.8), then $H_{\lambda, q, s}\left(\alpha_{1}\right) f(z) \prec q(z)$, and $q$ is the best dominant.

Proof. Similar usage of the arguments as in [6, Theorem 2.3e, page 31], we deduce that $q$ is a dominant from Theorem 2.3 and Theorem 2.4. Since $q$ satisfies (2.9), it is also a solution of (2.8) and therefore $q$ will be dominated by all dominants. Hence $q$ is the best dominant.

Now, we apply Theorem 2.2 to a specific case for $q(z)=$ $M z, M>0$.

In the particular case $q(z)=M z, M>0$, and in view of Definition 2.1, the class of admissible functions $\Phi_{H}[\Omega, q]$, denoted by $\Phi_{H}[\Omega, M]$, is described below.

Definition 2.6. Let $\Omega$ be a set in $\mathbb{C}$ and $M>0$. The class of admissible functions $\Phi_{H}[\Omega, M]$ consists of those functions $\phi: \mathbb{C}^{3} \times \mathbb{U} \times \overline{\mathbb{U}} \rightarrow \mathbb{C}$ such that

$$
\begin{aligned}
\phi\left(M e^{i \theta}, \frac{k+\alpha_{1}-2}{\alpha_{1}-1} M e^{i \theta},\right. \\
\left.\quad \frac{L+\left[\left(\alpha_{1}-2\right)\left(\alpha_{1}-3\right)+k\left(\alpha_{1}-4\right)\right] M e^{i \theta}}{\left(\alpha_{1}-1\right)\left(\alpha_{2}-1\right)}: z, \zeta\right) \notin \Omega,
\end{aligned}
$$

whenever $z \in \mathbb{U}, \theta \in \mathbb{R}$ and $\mathfrak{R}\left\{L e^{-i \theta}\right\} \geq(k-1) k M$ for all $\theta$, $\zeta \in \overline{\mathbb{U}}$ and $k \geq 1$.

Corollary 2.7. Let $\phi \in \Phi_{H}[\Omega, M]$. If $f \in \mathscr{A}$ satisfies

$$
\begin{array}{r}
\phi\left(H_{\lambda, q, s}\left(\alpha_{1}\right) f(z), H_{\lambda, q, s}\left(\alpha_{1}-1\right) f(z),\right. \\
\left.H_{\lambda, q, s}\left(\alpha_{1}-2\right) f(z) ; z, \zeta\right) \in \Omega
\end{array}
$$

then $\left|H_{\lambda, q, s}\left(\alpha_{1}\right) f(z)\right|<M$.

For the special case $\Omega=q(\mathbb{U})=\{w:|w|<M\}$, the class $\Phi_{H}[\Omega, M]$ is simply denoted by $\Phi_{H}[M]$.
Corollary 2.8. Let $\phi \in \Phi_{H}[M]$. If $f \in \mathscr{A}$ satisfies

$$
\begin{array}{r}
\mid \phi\left(H_{\lambda, q, s}\left(\alpha_{1}\right) f(z), H_{\lambda, q, s}\left(\alpha_{1}-1\right) f(z),\right. \\
\left.H_{\lambda, q, s}\left(\alpha_{1}-2\right) f(z) ; z, \zeta\right) \mid<M,
\end{array}
$$

then $\left|H_{\lambda, q, s}\left(\alpha_{1}\right) f(z)\right|<M$.

Definition 2.9. Let $\Omega$ be a set in $\mathbb{C}$ and $q \in \mathscr{Q}_{1} \mathscr{A} p \mathscr{H}$. The class of admissible function $\Phi_{H, 1}[\Omega, q]$ consists of those function $\phi: \mathbb{C}^{3} \times \mathbb{U} \times \overline{\mathbb{U}} \rightarrow \mathbb{C}$ that satisfy the admissibility condition: $\phi(u, v, w ; z, \zeta) \notin \Omega$ whenever $u=q(\xi)$, $v=\frac{1}{\alpha_{1}-2}\left(1+\left(\alpha_{1}-1\right) q(\xi)+\frac{k \xi q^{\prime}(\xi)}{q(\xi)}\right) \quad(q(\xi) \neq 0)$, and

$$
\begin{aligned}
& \Re\left\{\frac{\left[\alpha_{1}(w-u)+(2 v-3 w)+1\right]\left(\alpha_{1}-2\right) v}{\alpha_{1}(v-u)+(u-2 v)-1}\right. \\
& \left.+\alpha_{1}(v-2 u)+2(u-v)-1\right\} \geq k \Re\left\{\frac{\xi q^{\prime \prime}(\xi)}{q^{\prime}(\xi)}+1\right\},
\end{aligned}
$$

$(z \in \mathbb{U} ; \xi \in \partial \mathbb{U} \backslash E(q) ; \zeta \in \overline{\mathbb{U}} ; k \geq 1)$.

Theorem 2.10. Let $\phi \in \Phi_{H, 1}[\Omega, q]$. If $f \in \mathscr{A}$ satisfies

$$
\begin{aligned}
& \left\{\phi \left(\frac{H_{\lambda, q, s}\left(\alpha_{1}-1\right) f(z)}{H_{\lambda, q, s}\left(\alpha_{1}\right) f(z)}, \frac{H_{\lambda, q, s}\left(\alpha_{1}-2\right) f(z)}{H_{\lambda, q, s}\left(\alpha_{1}-1\right) f(z)},\right.\right. \\
& \left.\left.\frac{H_{\lambda, q, s}\left(\alpha_{1}-3\right) f(z)}{H_{\lambda, q, s}\left(\alpha_{1}-2\right) f(z)} ; z, \zeta\right): z \in \mathbb{U}, \zeta \in \bar{U}\right\} \subset \Omega,
\end{aligned}
$$

then $\frac{H_{\lambda, q, s}\left(\alpha_{1}-1\right) f(z)}{H_{\lambda, q, s}\left(\alpha_{1}\right) f(z)} \prec q(z)$.

Proof. Define the analytic function $p$ in $\mathbb{U}$ by

$$
p(z):=\frac{H_{\lambda, q, s}\left(\alpha_{1}-1\right) f(z)}{H_{\lambda, q, s}\left(\alpha_{1}\right) f(z)} .
$$

Using ((2.13)), we get

$$
\frac{z p^{\prime}(z)}{p(z)}:=\frac{z\left(H_{\lambda, q, s}\left(\alpha_{1}-1\right) f(z)\right)^{\prime}}{H_{\lambda, q, s}\left(\alpha_{1}-1\right) f(z)}-\frac{z\left(H_{\lambda, q, s}\left(\alpha_{1}\right) f(z)\right)^{\prime}}{H_{\lambda, q, s}\left(\alpha_{1}\right) f(z)} .
$$

By the use of (1.7) in (2.14), we get

$$
\frac{H_{\lambda, q, s}\left(\alpha_{1}-2\right) f(z)}{H_{\lambda, q, s}\left(\alpha_{1}-1\right) f(z)}=\frac{1}{\alpha_{1}-2}\left(\frac{z p^{\prime}(z)}{p(z)}+\left(\alpha_{1}-1\right) p(z)+1\right) \text {. }
$$

Further computations show that

$$
\begin{aligned}
& \frac{H_{\lambda, q, s}\left(\alpha_{1}-3\right) f(z)}{H_{\lambda, q, s}\left(\alpha_{1}-2\right) f(z)}=\frac{1}{\alpha_{1}-3}\left\{\left(\alpha_{1}-1\right) p(z)+\frac{z p^{\prime}(z)}{p(z)}\right. \\
& \left.+\frac{\left(\alpha_{1}-1\right) z p^{\prime}(z)+\frac{z p^{\prime}(z)}{p(z)}+\frac{z^{2} p^{\prime \prime}(z)}{p(z)}-\left(\frac{z p^{\prime}(z)}{p(z)}\right)^{2}}{1+\left(\alpha_{1}-1\right) p(z)+\frac{z p^{\prime}(z)}{p(z)}}\right\}
\end{aligned}
$$


We now define the transformations from $\mathbb{C}^{3}$ to $\mathbb{C}$ by

$$
u=r, v=\frac{1}{\alpha_{1}-2}\left(1+\left(\alpha_{1}-1\right) r+\frac{s}{r}\right),
$$

$$
w=\frac{1}{\alpha_{1}-3}\left\{\left(\alpha_{1}-1\right) r+\frac{s}{r}+\frac{\left(\alpha_{1}-1\right) s+\frac{t}{r}+\frac{s}{r}-\left(\frac{s}{r}\right)^{2}}{1+\left(\alpha_{1}-1\right) r+\frac{s}{r}}\right\} .
$$

Let $\psi(r, s, t ; z, \zeta)=\phi(u, v, w ; z, \zeta)$

$$
\begin{aligned}
& =\phi\left(r, \frac{1}{\alpha_{1}-2}\left(1+\left(\alpha_{1}-1\right) r+\frac{s}{r}\right),\right. \\
& \left.\frac{1}{\alpha_{1}-3}\left\{\left(\alpha_{1}-1\right) r+\frac{s}{r}+\frac{\left(\alpha_{1}-1\right) s+\frac{t}{r}+\frac{s}{r}-\left(\frac{s}{r}\right)^{2}}{1+\left(\alpha_{1}-1\right) r+\frac{s}{r}}\right\} ; z, \zeta\right) .
\end{aligned}
$$

The proof shall make use of Lemma 1.5. Using (2.13), (2.15), (2.17), from (2.18), we obtain

$$
\begin{aligned}
& \psi\left(p(z), z p^{\prime}(z), z^{2} p^{\prime \prime}(z) ; z, \zeta\right)=\phi\left(\frac{H_{\lambda, q, s}\left(\alpha_{1}-1\right) f(z)}{H_{\lambda, q, s}\left(\alpha_{1}\right) f(z)},\right. \\
& \left.\frac{H_{\lambda, q, s}\left(\alpha_{1}-2\right) f(z)}{H_{\lambda, q, s}\left(\alpha_{1}-1\right) f(z)}, \frac{H_{\lambda, q, s}\left(\alpha_{1}-3\right) f(z)}{H_{\lambda, q, s}\left(\alpha_{1}-2\right) f(z)} ; z, \zeta\right) .
\end{aligned}
$$

Hence (2.12) becomes $\psi\left(p(z), z p^{\prime}(z), z^{2} p^{\prime \prime}(z) ; z, \zeta\right) \in \Omega$. A computation using (2.17) yields

$$
\begin{gathered}
\frac{t}{s}+1=\frac{\left[\alpha_{1}(w-u)+(2 v-3 w)+1\right]\left(\alpha_{1}-2\right) v}{\alpha_{1}(v-u)+(u-2 v)-1} \\
+\alpha_{1}(v-2 u)+2(u-v)-1 .
\end{gathered}
$$

Thus the admissibility condition for $\phi \in \Phi_{H, 1}[\Omega, q]$ in Definition 2.9 is equivalent to the admissibility condition for $\psi$ as given in Definition 1.3. Hence $\psi \in \Psi[\Omega, q]$ and by Lemma $1.5 p(z) \prec q(z)$ or, equivalently, $\frac{H_{\lambda, q, s}\left(\alpha_{1}-1\right) f(z)}{H_{\lambda, q, s}\left(\alpha_{1}\right) f(z)} \prec q(z)$, which evidently completes the proof of Theorem 2.10.

If $\Omega \neq \mathbb{C}$ is a simply connected domain, then $\Omega=h(\mathbb{U})$ for some conformal mapping $h$ of $\mathbb{U}$ onto $\Omega$. In this case, the class $\Phi_{H, 1}[h(\mathbb{U}), q]$ is written as $\Phi_{H, 1}[h, q]$. The following result is an immediate consequence of Theorem 2.10.

Theorem 2.11. Let $\phi \in \Phi_{H, 1}[\Omega, q]$. If $f \in \mathscr{A}$ satisfies

$$
\begin{gathered}
\phi\left(\frac{H_{\lambda, q, s}\left(\alpha_{1}-1\right) f(z)}{H_{\lambda, q, s}\left(\alpha_{1}\right) f(z)}, \frac{H_{\lambda, q, s}\left(\alpha_{1}-2\right) f(z)}{H_{\lambda, q, s}\left(\alpha_{1}-1\right) f(z)},\right. \\
\left.\frac{H_{\lambda, q, s}\left(\alpha_{1}-3\right) f(z)}{H_{\lambda, q, s}\left(\alpha_{1}-2\right) f(z)} ; z, \zeta\right) \prec \prec h(z) \\
\text { then } \frac{H_{\lambda, q, s}\left(\alpha_{1}-1\right) f(z)}{H_{\lambda, q, s}\left(\alpha_{1}\right) f(z)} \prec q(z) .
\end{gathered}
$$

We will apply Theorem 2.10 to a specific case for $q(z)=$ $1+M z, M>0$.

In the particular case $q(z)=1+M z, M>0$, and in view of Definition 2.9, the class of admissible functions $\Phi_{H, 1}[\Omega, q]$, denoted by $\Phi_{H, 1}[\Omega, M]$, is described below.

Definition 2.12. Let $\Omega$ be a set in $\mathbb{C}$ and $M>0$. The class of admissible functions $\Phi_{H, 1}[\Omega, M]$ consists of those functions $\phi: \mathbb{C}^{3} \times \mathbb{U} \times \overline{\mathbb{U}} \rightarrow \mathbb{C}$ such that

$$
\begin{gathered}
\phi\left(1+M e^{i \theta}, \frac{1}{\left(\alpha_{1}-2\right)}\left[\alpha_{1}+\frac{k+\left(\alpha_{1}-1\right)\left(1+M e^{i \theta}\right)}{\left(1+M e^{i \theta}\right)} M e^{i \theta}\right],\right. \\
\frac{1}{\alpha_{1}-3}\left[\alpha_{1}-1+\frac{k+\left(\alpha_{1}-1\right)\left(1+M e^{i \theta}\right)}{\left(1+M e^{i \theta}\right)} M e^{i \theta}\right. \\
\left.+\frac{\left(M+e^{-i \theta}\right)\left[L e^{-i \theta}+k M\left(\alpha_{1}-1\right)\left(M+e^{-i \theta}\right)+k M\right]-k^{2} M^{2}}{\left(M+e^{-i \theta}\right)\left\{\left(M+e^{-i \theta}\right)\left[\alpha_{1}+\left(\alpha_{1}-1\right) M e^{i \theta}\right]\right\}}\right] \\
: z, \zeta) \notin \Omega,
\end{gathered}
$$

whenever $z \in \mathbb{U}, \theta \in \mathbb{R}$ and $\Re\left\{L e^{-i \theta}\right\} \geq(k-1) k M$ for all $\theta$, $\mu>0, \zeta \in \overline{\mathbb{U}}$ and $k \geq 1$.

Corollary 2.13. Let $\phi \in \Phi_{H, 1}[\Omega, M]$. If $f \in \mathscr{A}$ satisfies

$$
\begin{aligned}
\phi\left(\frac{H_{\lambda, q, s}\left(\alpha_{1}-1\right) f(z)}{H_{\lambda, q, s}\left(\alpha_{1}\right) f(z)}, \frac{H_{\lambda, q, s}\left(\alpha_{1}-2\right) f(z)}{H_{\lambda, q, s}\left(\alpha_{1}-1\right) f(z)},\right. \\
\left.\frac{H_{\lambda, q, s}\left(\alpha_{1}-3\right) f(z)}{H_{\lambda, q, s}\left(\alpha_{1}-2\right) f(z)} ; z, \zeta\right) \in \Omega,
\end{aligned}
$$

then $\left|\frac{H_{\lambda, q, s}\left(\alpha_{1}-1\right) f(z)}{H_{\lambda, q, s}\left(\alpha_{1}\right) f(z)}-1\right|<M$.

For the special case $\Omega=q(\mathbb{U})=\{w:|w-1|<M\}$, the class $\Phi_{H, 1}[\Omega, M]$ is simply denoted by $\Phi_{H, 1}[M]$.

Corollary 2.14. Let $\phi \in \Phi_{H, 1}[M]$. If $f \in \mathscr{A}$ satisfies

$$
\begin{aligned}
\mid \phi\left(\frac{H_{\lambda, q, s}\left(\alpha_{1}-1\right) f(z)}{H_{\lambda, q, s}\left(\alpha_{1}\right) f(z)}, \frac{H_{\lambda, q, s}\left(\alpha_{1}-2\right) f(z)}{H_{\lambda, q, s}\left(\alpha_{1}-1\right) f(z)},\right. \\
\left.\frac{H_{\lambda, q, s}\left(\alpha_{1}-3\right) f(z)}{H_{\lambda, q, s}\left(\alpha_{1}-2\right) f(z)} ; z, \zeta\right)-1 \mid<M,
\end{aligned}
$$

then $\left|\frac{H_{\lambda, q, s}\left(\alpha_{1}-1\right) f(z)}{H_{\lambda, q, s}\left(\alpha_{1}\right) f(z)}-1\right|<M$.

\section{Superordination and Sandwich-type Results}

In this section, we investigate the dual problem of strong differential subordination (that is, strong differential superordination). For this purpose, the class of admissible functions is given in the following definition. 
Definition 3.1. Let $\Omega$ be a set in $\mathbb{C}, q \in \mathscr{H}$ with $q^{\prime}(z) \neq 0$. The class of admissible functions $\Phi_{H}^{\prime}[\Omega, q]$ consists of those functions $\phi: \mathbb{C}^{3} \times \mathbb{U} \times \overline{\mathbb{U}} \rightarrow \mathbb{C}$ that satisfy the admissibility condition: $\phi(u, v, w ; \xi, \zeta) \in \Omega$ whenever $u=q(z)$, $v=\frac{z q^{\prime}(z)+m\left(\alpha_{1}-2\right) q(z)}{m\left(\alpha_{1}-1\right)}$, and

$$
\begin{gathered}
\Re\left\{\frac{\left(\alpha_{1}-1\right)\left(\alpha_{2}-1\right) w-\left(\alpha_{1}-2\right)\left(\alpha_{1}-3\right) u}{\left(\alpha_{1}-1\right) v-(} \alpha_{1}-2\right) u \\
\leq \frac{1}{m} \Re\left\{\frac{z q^{\prime \prime}(z)}{q^{\prime}(z)}+1\right\},
\end{gathered}
$$

$(z \in \mathbb{U} ; \xi \in \partial \mathbb{U} ; \zeta \in \overline{\mathbb{U}} ; m \geq 1)$.

Theorem 3.2. Let $\phi \in \Phi_{H}^{\prime}[\Omega, q] . \quad$ If $f \in \mathscr{A}$, $H_{\lambda, q, s}\left(\alpha_{1}\right) f(z) \in \mathscr{Q}_{0}$ and

$\phi\left(H_{\lambda, q, s}\left(\alpha_{1}\right) f(z), H_{\lambda, q, s}\left(\alpha_{1}-1\right) f(z), H_{\lambda, q, s}\left(\alpha_{1}-2\right) f(z) ; z, \zeta\right)$ is univalent in $\mathbb{U}$, then

$$
\begin{gathered}
\Omega \subset\left\{\phi \left(H_{\lambda, q, s}\left(\alpha_{1}\right) f(z), H_{\lambda, q, s}\left(\alpha_{1}-1\right) f(z),\right.\right. \\
\left.\left.H_{\lambda, q, s}\left(\alpha_{1}-2\right) f(z) ; z, \zeta\right): z, \zeta\right\}
\end{gathered}
$$

implies

$$
q(z) \prec H_{\lambda, q, s}\left(\alpha_{1}\right) f(z) .
$$

Proof. With $p(z)=H_{\lambda, q, s}\left(\alpha_{1}\right) f(z)$ and

$\psi(r, s, t ; z, \zeta)$

$=\phi\left(r, \frac{s+\left(\alpha_{1}-2\right) r}{\alpha_{1}-1}, \frac{t+\left(\alpha_{1}-4\right) s+\left(\alpha_{1}-2\right)\left(\alpha_{1}-3\right) r}{\left(\alpha_{1}-1\right)\left(\alpha_{2}-1\right)} ; \xi, \zeta\right)$

$=\phi(u, v, w ; \xi, \zeta)$,

equation (2.7) and (3.1) yields

$$
\Omega \subset\left\{\psi\left(p(z), z p^{\prime}(z), z^{2} p^{\prime \prime}(z) ; z, \zeta\right): z \in \mathbb{U}, \zeta \in \overline{\mathbb{U}}\right\} .
$$

Since

$$
\frac{t}{s}+1=\frac{\left(\alpha_{1}-1\right)\left(\alpha_{2}-1\right) w-\left(\alpha_{1}-2\right)\left(\alpha_{1}-3\right) u}{\left(\alpha_{1}-1\right) v-\left(\alpha_{1}-2\right) u}-\alpha_{1}+5,
$$

the admissibility condition for $\phi \in \Phi_{H}^{\prime}[\Omega, q]$ in Definition 3.1 is equivalent to the admissibility condition for $\psi$ as given in Definition 1.4. Hence $\psi \in \Psi^{\prime}[\Omega, q]$, and by Lemma 1.6 $q(z) \prec p(z)$ or $q(z) \prec H_{\lambda, q, s}\left(\alpha_{1}\right) f(z)$.

If $\Omega \neq \mathbb{C}$ is a simply connected domain, the $\Omega=h(\mathbb{U})$ for some conformal mapping $h$ of $\mathbb{U}$ onto $\Omega$. In this case, the class $\Phi_{H}^{\prime}[h(\mathbb{U}), q]$ is written as $\Phi_{H}^{\prime}[h, q]$, Theorem 3.2 can be written in the following form.

Theorem 3.3. Let $\phi \in \Phi_{H}^{\prime}[h, q]$. Let $q \in \mathscr{H}$ and $h$ be analytic in $\mathbb{U}$. If $f \in \mathscr{A}, H_{\lambda, q, s}\left(\alpha_{1}\right) f(z) \in \mathscr{Q}_{0}$ and

$\phi\left(H_{\lambda, q, s}\left(\alpha_{1}\right) f(z), H_{\lambda, q, s}\left(\alpha_{1}-1\right) f(z), H_{\lambda, q, s}\left(\alpha_{1}-2\right) f(z) ; z, \zeta\right)$ is univalent in $\mathbb{U}$, then

$$
\begin{gathered}
h(z) \prec \prec \phi\left(H_{\lambda, q, s}\left(\alpha_{1}\right) f(z), H_{\lambda, q, s}\left(\alpha_{1}-1\right) f(z),\right. \\
\left.H_{\lambda, q, s}\left(\alpha_{1}-2\right) f(z) ; z, \zeta\right)
\end{gathered}
$$

implies $q(z) \prec H_{\lambda, q, s}\left(\alpha_{1}\right) f(z)$.
Theorem 3.2 and Theorem 3.3 can only be used to obtain subordinants of differential superordination of the form (3.1) or (3.3). The following theorem proves the existence of the best subordinant of (3.3) for an appropriate $\phi$.

Theorem 3.4. Let $h$ be analytic in $\mathbb{U}$ and $\phi: \mathbb{C}^{3} \times \mathbb{U} \times \overline{\mathbb{U}} \rightarrow \mathbb{C}$. Suppose that the differential equation

$$
\begin{aligned}
& \phi\left(q(z), \frac{z q^{\prime}(z)+\left(\alpha_{1}-2\right) q(z)}{\alpha_{1}-1},\right. \\
& \left.\frac{z^{2} q^{\prime \prime}(z)+\left(\alpha_{1}-4\right) z q^{\prime}(z)+\left(\alpha_{1}-2\right)\left(\alpha_{1}-3\right) q(z)}{\left(\alpha_{1}-1\right)\left(\alpha_{2}-1\right)} ; z, \zeta\right)=h(z)
\end{aligned}
$$

has a solution $q \in \mathscr{Q}_{0}$. If $\phi \in \Phi_{H}^{\prime}[h, q], f \in \mathscr{A}$, $H_{\lambda, q, s}\left(\alpha_{1}\right) f(z) \in \mathscr{Q}_{0}$ and

$\phi\left(H_{\lambda, q, s}\left(\alpha_{1}\right) f(z), H_{\lambda, q, s}\left(\alpha_{1}-1\right) f(z), H_{\lambda, q, s}\left(\alpha_{1}-2\right) f(z) ; z, \zeta\right)$ is univalent in $\mathbb{U}$, then

$$
\begin{array}{r}
h(z) \prec \prec \phi\left(H_{\lambda, q, s}\left(\alpha_{1}\right) f(z), H_{\lambda, q, s}\left(\alpha_{1}-1\right) f(z),\right. \\
\left.H_{\lambda, q, s}\left(\alpha_{1}-2\right) f(z) ; z, \zeta\right)
\end{array}
$$

implies $q(z) \prec H_{\lambda, q, s}\left(\alpha_{1}\right) f(z)$ and $q$ is the best subordinant.

Proof. The proof is similar to that of Theorem 2.5, and so it is being omitted here.

By combining Theorem 2.3 and Theorem 3.3 , we obtain the following sandwich-type theorem.

Corollary 3.5. Let $h_{1}$ and $q_{1}$ be analytic functions in $\mathbb{U}, h_{2}$ be univalent function in $\mathbb{U}, q_{2} \in \mathscr{Q}_{0}$ with $q_{1}(0)=q_{2}(0)=0$ and $\phi \in \Phi_{H}\left[h_{2}, q_{2}\right] \cap \Phi_{H}^{\prime}\left[h_{1}, q_{1}\right]$. If $f \in \mathscr{A}, H_{\lambda, q, s}\left(\alpha_{1}\right) f(z) \in$ $\mathscr{H} \cap \mathscr{Q}_{0}$ and

$\phi\left(H_{\lambda, q, s}\left(\alpha_{1}\right) f(z), H_{\lambda, q, s}\left(\alpha_{1}-1\right) f(z), H_{\lambda, q, s}\left(\alpha_{1}-2\right) f(z) ; z, \zeta\right)$ is univalent in $\mathbb{U}$, then

$$
\begin{aligned}
h_{1}(z) \prec \prec & \\
\phi\left(H_{\lambda, q, s}\left(\alpha_{1}\right) f(z), H_{\lambda, q, s}\left(\alpha_{1}-1\right) f(z), H_{\lambda, q, s}\right. & \left.\left.\alpha_{1}-2\right) f(z) ; z, \zeta\right) \\
& \prec \prec h_{2}(z)
\end{aligned}
$$

implies $q_{1}(z) \prec H_{\lambda, q, s}\left(\alpha_{1}\right) f(z) \prec q_{2}(z)$.

Definition 3.6. Let $\Omega$ be a set in $\mathbb{C}, q \in \mathscr{Q}_{1} \cap \mathscr{H}$ with $q(z) \neq 0$. The class of admissible function $\Phi_{H, 1}^{\prime}[\Omega, q]$ consists of those function $\phi: \mathbb{C}^{3} \times \mathbb{U} \times \overline{\mathbb{U}} \rightarrow \mathbb{C}$ that satisfy the admissibility condition: $\phi(u, v, w ; \xi, \zeta) \in \Omega$ whenever

$$
u=q(z), \quad v=\frac{1}{\alpha_{1}-2}\left(1+\left(\alpha_{1}-1\right) q(z)+\frac{z q^{\prime}(z)}{m q(z)}\right),
$$

and

$$
\begin{array}{r}
\Re\left\{\frac{\left[\alpha_{1}(w-u)+(2 v-3 w)+1\right]\left(\alpha_{1}-2\right) v}{\alpha_{1}(v-u)+(u-2 v)-1}+\alpha_{1}(v-2 u)\right. \\
+2(u-v)-1\} \geq \frac{1}{m} \Re\left\{\frac{z q^{\prime \prime}(z)}{q^{\prime}(z)}+1\right\},
\end{array}
$$

$(z \in \mathbb{U} ; \xi \in \partial \mathbb{U} ; \zeta \in \overline{\mathbb{U}} ; m \geq 1)$. 
Theorem 3.7. Let $\phi \in \Phi_{H, 1}^{\prime}[\Omega, q]$. If $f \in \mathscr{A}, \frac{H_{\lambda, q, s}\left(\alpha_{1}-1\right) f(z)}{H_{\lambda, q, s}\left(\alpha_{1}\right) f(z)} \in$ $\mathscr{Q}_{1}$ and

$$
\begin{gathered}
\phi\left(\frac{H_{\lambda, q, s}\left(\alpha_{1}-1\right) f(z)}{H_{\lambda, q, s}\left(\alpha_{1}\right) f(z)}, \frac{H_{\lambda, q, s}\left(\alpha_{1}-2\right) f(z)}{H_{\lambda, q, s}\left(\alpha_{1}-1\right) f(z)},\right. \\
\left.\frac{H_{\lambda, q, s}\left(\alpha_{1}-3\right) f(z)}{H_{\lambda, q, s}\left(\alpha_{1}-2\right) f(z)} ; z, \zeta\right)
\end{gathered}
$$

is univalent in $\mathbb{U}$, then

$$
\begin{gathered}
\Omega \subset\left\{\phi \left(\frac{H_{\lambda, q, s}\left(\alpha_{1}-1\right) f(z)}{H_{\lambda, q, s}\left(\alpha_{1}\right) f(z)}, \frac{H_{\lambda, q, s}\left(\alpha_{1}-2\right) f(z)}{H_{\lambda, q, s}\left(\alpha_{1}-1\right) f(z)},\right.\right. \\
\left.\left.\frac{H_{\lambda, q, s}\left(\alpha_{1}-3\right) f(z)}{H_{\lambda, q, s}\left(\alpha_{1}-2\right) f(z)} ; z, \zeta\right): z, \zeta\right\}
\end{gathered}
$$

implies

$$
q(z) \prec \frac{H_{\lambda, q, s}\left(\alpha_{1}-1\right) f(z)}{H_{\lambda, q, s}\left(\alpha_{1}\right) f(z)} .
$$

Proof. From (2.19) and (3.7), we have

$$
\Omega \subset\left\{\psi\left(p(z), z p^{\prime}(z), z^{2} p^{\prime \prime}(z) ; z, \zeta\right): z \in \mathbb{U}, \zeta \in \overline{\mathbb{U}}\right\} .
$$

In view of (2.17), the admissibility condition for $\phi \in \Phi_{H, 1}^{\prime}[\Omega, q]$ in Definition 3.6 is equivalent to the admissibility condition for $\psi$ as given in Definition 1.4. Hence $\psi \in \Psi^{\prime}[\Omega, q]$, and by Lemma $1.6 q(z) \prec p(z)$ or $q(z) \prec \frac{H_{\lambda, q, s}\left(\alpha_{1}-1\right) f(z)}{H_{\lambda, q, s}\left(\alpha_{1}\right) f(z)}$.

If $\Omega \neq \mathbb{C}$ is a simply connected domain, the $\Omega=h(\mathbb{U})$ for some conformal mapping $h$ of $\mathbb{U}$ onto $\Omega$. In this case, the class $\Phi_{H, 1}^{\prime}[h(\mathbb{U}), q]$ is written as $\Phi_{H, 1}^{\prime}[h, q]$. Proceeding similarly as in the previous section, the following result is an immediate consequence of Theorem 3.7.

Theorem 3.8. Let $\phi \in \Phi_{H, 1}^{\prime}[h, q]$. Let $q \in \mathscr{H}$, and $h$ be analytic in $\mathbb{U}$. If $f \in \mathscr{A}, \frac{H_{\lambda, q, s}\left(\alpha_{1}-1\right) f(z)}{H_{\lambda, q, s}\left(\alpha_{1}\right) f(z)} \in \mathscr{Q}_{1}$ and

$$
\begin{aligned}
\phi\left(\frac{H_{\lambda, q, s}\left(\alpha_{1}-1\right) f(z)}{H_{\lambda, q, s}\left(\alpha_{1}\right) f(z)}, \frac{H_{\lambda, q, s}\left(\alpha_{1}-2\right) f(z)}{H_{\lambda, q, s}\left(\alpha_{1}-1\right) f(z)},\right. \\
\left.\frac{H_{\lambda, q, s}\left(\alpha_{1}-3\right) f(z)}{H_{\lambda, q, s}\left(\alpha_{1}-2\right) f(z)} ; z, \zeta\right)
\end{aligned}
$$

is univalent in $\mathbb{U}$, then

$$
\begin{aligned}
h(z) \prec \prec \phi( & \frac{H_{\lambda, q, s}\left(\alpha_{1}-1\right) f(z)}{H_{\lambda, q, s}\left(\alpha_{1}\right) f(z)}, \frac{H_{\lambda, q, s}\left(\alpha_{1}-2\right) f(z)}{H_{\lambda, q, s}\left(\alpha_{1}-1\right) f(z)}, \\
& \left.\frac{H_{\lambda, q, s}\left(\alpha_{1}-3\right) f(z)}{H_{\lambda, q, s}\left(\alpha_{1}-2\right) f(z)} ; z, \zeta\right)
\end{aligned}
$$

implies $q(z) \prec \frac{H_{\lambda, q, s}\left(\alpha_{1}-1\right) f(z)}{H_{\lambda, q, s}\left(\alpha_{1}\right) f(z)}$.
By combining Theorem 2.11 and Theorem 3.8 we obtain the following sandwich-type theorem.

Corollary 3.9. Let $h_{1}$ and $q_{1}$ be analytic functions in $\mathbb{U}, h_{2}$ be univalent function in $\mathbb{U}, q_{2} \in \mathscr{Q}_{1}$ with $q_{1}(0)=q_{2}(0)=1$ and $\phi \in \Phi_{H, 1}\left[h_{2}, q_{2}\right] \cap \Phi_{H, 1}^{\prime}\left[h_{1}, q_{1}\right]$. If $f \in \mathscr{A}, \frac{H_{\lambda, q, s}\left(\alpha_{1}-1\right) f(z)}{H_{\lambda, q, s}\left(\alpha_{1}\right) f(z)} \in$ $\mathscr{H} \cap \mathscr{Q}_{1}$ and

$$
\begin{aligned}
\phi\left(\frac{H_{\lambda, q, s}\left(\alpha_{1}-1\right) f(z)}{H_{\lambda, q, s}\left(\alpha_{1}\right) f(z)}, \frac{H_{\lambda, q, s}\left(\alpha_{1}-2\right) f(z)}{H_{\lambda, q, s}\left(\alpha_{1}-1\right) f(z)},\right. \\
\left.\frac{H_{\lambda, q, s}\left(\alpha_{1}-3\right) f(z)}{H_{\lambda, q, s}\left(\alpha_{1}-2\right) f(z)} ; z, \zeta\right)
\end{aligned}
$$

is univalent in $\mathbb{U}$, then

$$
\begin{array}{r}
h_{1}(z) \prec \prec \phi\left(\frac{H_{\lambda, q, s}\left(\alpha_{1}-1\right) f(z)}{H_{\lambda, q, s}\left(\alpha_{1}\right) f(z)}, \frac{H_{\lambda, q, s}\left(\alpha_{1}-2\right) f(z)}{H_{\lambda, q, s}\left(\alpha_{1}-1\right) f(z)},\right. \\
\left.\frac{H_{\lambda, q, s}\left(\alpha_{1}-3\right) f(z)}{H_{\lambda, q, s}\left(\alpha_{1}-2\right) f(z)} ; z, \zeta\right) \prec \prec h_{2}(z)
\end{array}
$$

implies $q_{1}(z) \prec \frac{H_{\lambda, q, s}\left(\alpha_{1}-1\right) f(z)}{H_{\lambda, q, s}\left(\alpha_{1}\right) f(z)} \prec q_{2}(z)$.

\section{References}

[1] J. A. Antonino and S. Romaguera, Strong differential subordination to Briot-Bouquet differential equations, $J$. Differential Equations, (114) (1) (1994), 101-105.

[2] J. A. Antonino, Strong differential subordination and applications to univalency conditions, J. Korean Math. Soc. , (43) (2) (2006), 311-322.

[3] N. E. Cho, Oh Sang Kwon and H. M. Srivastava, Strong differential subordination and superordination for multivalently meromorphic functions involving the LiuSrivastava operator, Integral transforms and Spec. Funct., (21) (8) (2010), 589-601.

[4] J. H. Choi, M. Saigo and H. M. Srivastava, Some inclusion properties of a certain family of integral operators, J. Math. Anal. Appl., (276) (2002), 432-435.

[5] M. P. Jeyaraman and T. K. Suresh, Strong differential subordination and superordination of analytic functions, J. Math. Anal. Appl., (385) (2012), 854-864.

[6] S. S. Miller and P. T. Mocanu, Differential Subordination : Theory and Applications, Series on Monographs and Textbooks in Pure and Applied Mathematics (No. 225), Marcel Dekker, New York and Basel, 2000.

[7] S. S. Miller and P. T. Mocanu, Subordinants of differential superordinations, Complex Var. Theory Appl., (48) (10) (2003), 815-826.

[8] K. I. Noor, On new classes of integral operators, J. Nat. Geometry, (16) (1999), 71-80.

[9] G. I. Oros, First order strong differential superordination, Gen. Math., (15) (2-3) (2007), 77-87.

[10] G. I. Oros, Strong differential superordination, Acta Univ. Apulensis Math. Inform., (19) (2009), 101-106. 
[11] G. I. Oros, Sufficient conditions for univalence obtained by using second order linear strong differential subordinations, Turkish J. Math., (34) (2010), 13-20.

[12] G. I. Oros and G. Oros, First order linear strong differential subordination, Gen. Math., (15) (2-3)(2007), 98-107.

[13] G. I. Oros and G. Oros, Strong differential subordination, Turkish J. Math., (33) (2009), 249-257.

[14] Oh Sang Kwon and Nak Eun Cho, "Inclusion Properties for Certain Subclasses of Analytic Functions Associated with the Dziok-Srivastava Operator, J. Inequal. Appl., vol. 2007, Article ID 051079, 10 pages, 2007. doi:10.1155/2007/51079.

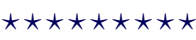

$\operatorname{ISSN}(\mathrm{P}): 2319-3786$

Malaya Journal of Matematik

$\operatorname{ISSN}(\mathrm{O}): 2321-5666$

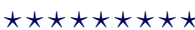

